By observing that neither the "sign" of $A_{0}$ nor that of $B_{0}$ enters into the proof, we see that we can diagonalize two semi-definite matrices (possibly of opposite sign). We can also easily extend the theorem to Hermitian matrices. Thus let a superscript asterisk denote complex conjugation and let $A$ and $B$ be complex Hermitian, positive semidefinite matrices. Proceeding as above, but using complex $T_{0}, T_{1}$ and unitary $T_{a}$, we can write

$$
\begin{aligned}
& A=T^{t^{*}} A_{o} T \\
& B=T^{t^{*}} B_{o} T
\end{aligned}
$$

where $A_{0}$ and $B_{0}$ are as in Eqs. (3) and (8).

3. Applications. The above theorem is necessary for the synthesis of networks which are passive or active at a point (to be published, for the basic concepts see [2]). It also can be used to advantage in the synthesis of two element kind networks, as well as in studying equivalent networks (see pp. 96 and 142 of [3]). Its use in studying the vibraticns of systems satisfying Lagrange's equations should also be apparent.

\title{
REFERENCES
}

1. H. Turnbull and A. Aitken, "An Introduction to the Theory of Canonical Matrices," Blackie \& Son 1952 , p. 107.

2. C. Desoer and E. Kuh, "Bounds on Natural Frequencies of Linear Active Networks," Proceedings of the Brooklyn Polytechnic Symposium on Active Networks and Feedback Systems, (1960).

3. E. A. Guillemin, "Synthesis of Passive Networks," John Wiley \& Sons, Inc., New York, 1957.

\section{AN UPPER BOUND ON RIGHT HALF PLANE ZEROS*}

\section{By DOV HAZONY (Case Institute of Technology)}

Abstract. An upper bound is placed on the number of right half plane zeros of functions of the type $Z-m / n . Z$ and $m / n$ are RLC and LC driving point impedance functions respectively. In addition, it is shown that if $\operatorname{Re} Z>0$ on $j$ axis, the number of right half plane zeros is determined precisely.

Introduction. In problems of control and network synthesis, it may be necessary to determine the number of right half plane zeros of certain impedance functions. In control problems, zeros in the right half plane may cause instability while in synthesis they may require active networks. In this paper an upper bound is placed on the number of these zeros of the class of functions $Z-m / n$ and $Z-n / m$. These terms are defined below.

\section{Lemma.}

Given: I. $\quad Z$ is prf (an RLC driving point impedance function).

II. $m+n$ is a Hurwitz polynomial, of degree $d$, of the complex variable $S ; m$ is an even and $n$ is an odd function of $S$.

${ }^{*}$ Received July 27, 1960. This paper, although based on the work sponsored by the U. S. Air Force, Cambridge Research Center, Bedford, Mass. Contract No. AF 19(604)3887, has not been approved or disapproved by that agency. 
IIIa. The real part of $Z, \operatorname{Re} Z$, may have some isolated zeros on the $j$ axis but it cannot be identically zero along any segment of the $j$ axis. (Part 1).

or IIIb. $\operatorname{Re} Z>0$ on $j$ axis. (Part 2).

Then $m z-n$ and $m-n z$ have, at most, $d$ zeros each in the right half $S$ plane(Part 1) and exactly $d$ zeros if IIIb is exposed (Part 2).

Part 1. Proof. Let $\zeta$ be given by

$$
\zeta=(m Z-n) /(m-n Z)
$$

from which follows

$$
\left(\frac{Z-1}{Z+1}\right)=\left(\frac{m-n}{m+n}\right)\left(\frac{\zeta-1}{\zeta+1}\right)
$$

Since $Z$ is prf (by I) and $|(m-n) /(m+n)|=i 1$ on the axis (by II)

$$
\left|\frac{Z-1}{Z+1}\right|=\left|\frac{\zeta-1}{\zeta+1}\right| \leq 1 \quad \text { on the } j \text { axis. }
$$

Let

$$
w=\frac{(Z-1) /(Z+1)}{(m-n) /(m+n)}=\frac{\zeta-1}{\zeta+1} \text { and } \quad \therefore|w| \leq 1 \quad \text { on } j \text { axis. }
$$

To prove the lemma, it will suffice to show that $\zeta$ has as many pole zero pairs in the right half plane as $w$ has poles, since $w$ may not have more than $d$ poles in the right half plane. From (4) the following can be verified

$$
\begin{aligned}
& \operatorname{Re} \zeta>0 \text { for }|w|<1 \\
& \operatorname{Re} \zeta=0 \text { for }|w|=1 \\
& \operatorname{Re} \zeta<0 \text { for }|w|>1
\end{aligned}
$$

Let $D$ be any $S$ plane domain such that (Fig. 1)

$$
\begin{aligned}
& |w|=1 \quad \text { along the boundaries of } D, \\
& |w|>1 \quad \text { inside } D .
\end{aligned}
$$

Then, by the maximum modulus theorem, $w$ has at least one pole inside $D$. Let $\zeta$ be given by $|\zeta| e^{i \alpha}$. Then by (5) and (6)

$$
\begin{aligned}
& |\alpha|=90^{\circ} \text { along the boundary of } D, \\
& |\alpha|>90^{\circ} \text { inside } D, \\
& |\alpha|<90^{\circ} \text { outside } D .
\end{aligned}
$$

Now, since the $90^{\circ}$ phase locus must start at a zero and end at a pole, it follows that all poles and zeros of $\zeta$ lie on the boundary of $D$ and occur in pairs. Further, any phase locus starts at a zero and ends at a pole. Then, inside $D, \zeta$ has as many $180^{\circ}$ phase loci as the number of pole zero pairs it has on the boundary of $D$. Along each $180^{\circ}$ phase loci the magnitude of $\zeta$ increases monotonically* from 0 to $\infty$. Then, at one point along this

\footnotetext{
*This is due to the orthogonal properties of magnitude and phase plots.
} 


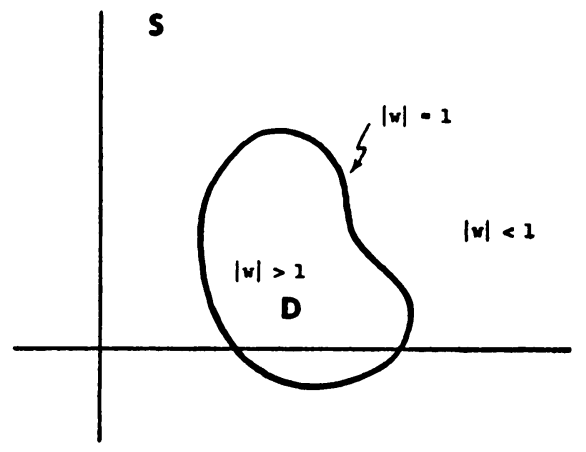

FIG. 1 A plot of $w$ in the $S$ plane.

line, $\zeta=-1$ and $w$ has a pole. It follows that $\zeta$ has as many pole zero pairs on the boundary of $D$ as the number of poles $w$ has inside. In addition, the following properties of $w$ in the right half $S$ plane may be ascertained:

$w$ has $d$ poles at most. (by (4))

If $w$ has poles it also has at least one domain $D$.

This $D$ must lie exclusively in the right half plane.

(by postulate III and because $|w| \leq 1$ on the $j$ axis, by (4))

It follows that $\zeta$ has $d$ poles and $d$ zeros in the right half $S$ plane at most. This completes the proof (Part 1).

Part 2. Rouche's theorem [1] will be used to prove Part 2.

Rouche's theorem. If $P(s)$ and $Q(s)$ are analytic interior to a simple closed Jordan curve $C$ and if they are continuous on $C$ and

$$
|P(s)|<|Q(s)| \text { on } C
$$

then the function $F(s)=P(s)+Q(s)$ has the same number of zeros interior to $C$ as does $Q(s)$.

Let $Q(s)=\frac{1}{2}(n-m) /(m+n), Q(s)$ has $d$ right half plane zeros and

$$
|Q(s)|=\frac{1}{2} \text { on } j \text { axis (by II). }
$$

and $\quad P(s)=\frac{1}{2}(Z-1) /(Z+1) . \quad|P(s)|<\frac{1}{2}$ on $j$ axis as $\operatorname{Re} Z>0$ there from which it follows that $F(s)=(n Z-m) /(m+n)(Z+1)$ and therefore also $n Z-m$ have $d$ right half plane zeros. Similarly, by letting $Q(s)=\frac{1}{2}(m-n) /(m+n), m Z-n$ has $d$ right half plane zeros.

This completes the proof.

Applications. Example 1. In the following network (Fig. 2) $Z=\operatorname{prf}, m / n=a$ reactance function $(m+n$ is of degree $d)$. Then, the transfer function,

$$
Z_{21}=\left.\frac{E_{2}}{E_{1}}\right|_{I_{2}=0}=\frac{n Z-m}{n Z+m}
$$

has, at most, $d$ right half plane zeros. 


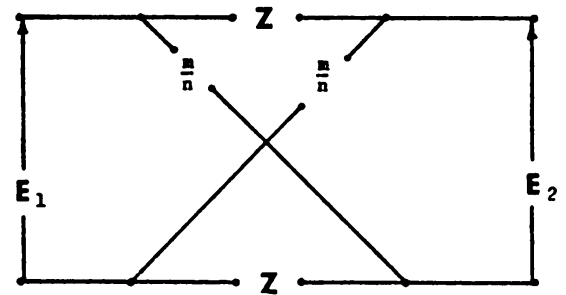

FIG. 2 A lattice in which $\left.\frac{E_{2}}{\bar{E}_{1}}\right|_{I_{2}-0}=\frac{n Z-m}{n Z+m}$.

Example 2. In the following network [2] (Fig. 3) $\zeta$ is given by ( $Z$ is prf)

$$
\begin{aligned}
\zeta=\left[\left(1+\frac{S^{2}}{a b} \frac{b Z(a)-a Z(b)}{b Z(b)-a Z(a)}\right) Z-S \frac{Z(a) Z(b)}{a b} \frac{b^{2}-a^{2}}{b Z(b)-a Z(a)}\right] \\
\cdot\left[\left(1+\frac{S^{2}}{a b} \frac{b Z(b)-a Z(a)}{b Z(a)-a Z(b)}\right)-S \frac{Z}{a b} \frac{b^{2}-a^{2}}{b Z(a)-a Z(b)}\right]^{-1}
\end{aligned}
$$

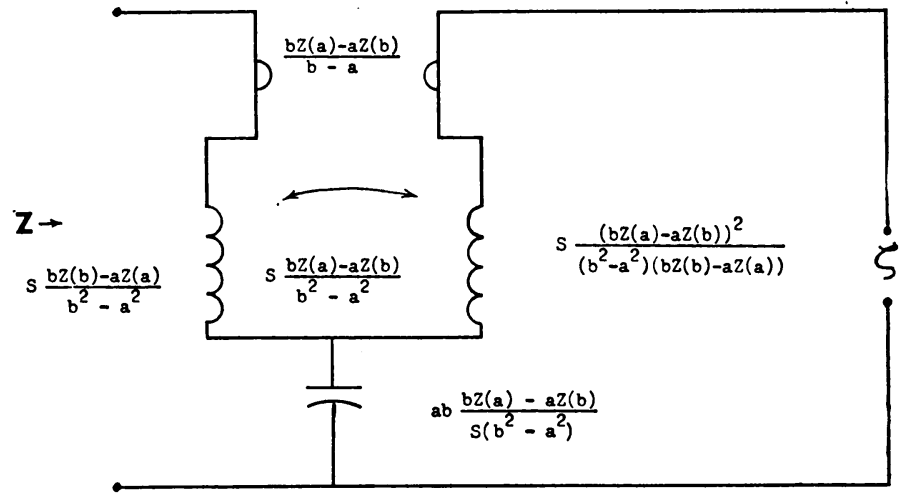

FIg. 3 Physical realization of an impedance function, $Z$, in terms of $\zeta$.

where $a$ and $b$ are positive real or complex conjugates with a positive real part. $\zeta$ is constructed to have the factor $(s-a)(s-b)$ common in both the numerator and denominator. Further, [2] $\operatorname{Re} \zeta \geq 0$ on the $j$ axis. To complete the proof of the realizability of $\zeta$ it is necessary to show that no additional right half plane zeros and poles are present. By the above lemma, both numerator and denominator have only two right half plane zeros each. This completes the proof.

\section{REFERENCES}

1. M. Marden, The geometry of the zeros of a polynomial in a complex variable, The American Mathematical Society, New York, 1949, Chap. 1

2. D. Hazony, Zero cancellation synthesis using impedance operators, to be published in an early issue of the IRE PGCT Transactions on Circuit Theory 